\title{
Publisher's Erratum \\ Erratum to: Existence and Regularity of Steady Flows for Shear-Thinning Liquids in Exterior Two-Dimensional Domains
}

\author{
Giovanni P. Galdi \& Carlo R. Grisanti
}

Erratum to: Arch. Rational. Mech. Anal. (2010) DOI 10.1007/s00205-010-0364-0

Unfortunately the article was published with the wrong title due to a typesetting error. The last word in the title was omitted.

The correct title of the article should read:

Existence and Regularity of Steady Flows for Shear-Thinning Liquids in Exterior Two-Dimensional Domains

\author{
University of Pittsburgh, \\ 630 Benedum Engineering Hall, \\ Pittsburgh, PA 15621, USA. \\ e-mail: galdi@pitt.edu \\ and \\ Dipartimento di Matematica Applicata "U. Dini”, \\ Università di Pisa, \\ Via Buonarroti, 1/c, 56127 Pisa, Italy. \\ e-mail: grisanti@dma.unipi.it
}

Published online January 5, 2011 - (C) Springer-Verlag (2010)

The online version of the original article can be found under doi:10.1007/s00205-0100364-0. 